\title{
Revista de Enfermagem
}

Rev enferm UFPE on line.

2021; $15: \mathrm{e} 247900$

DOI: $10.5205 / 1981-$

8963.2021.247900

https://periodicos.ufpe.br/r

evistas/revistaenfermagem

\section{PERFIL DE TRABALHADORES ACOMETIDOS POR TRANSTORNOS MENTAIS RELACIONADOS AO TRABALHO \\ PROFILE OF WORKERS AFFECTED BY WORK-RELATED MENTAL DISORDERS PERFIL DE LOS TRABAJADORES AFECTADOS POR TRASTORNOS MENTALES RELACIONADOS CON EL TRABAJO}

Adriana dos Santos Gomes ${ }^{1}$ (D) Adrian Thaís Cardoso Santos Gomes da Silva ${ }^{2}$, Ana Carla Paiva de Moura ${ }^{3}$, Danilo Martins Roque Pereira ${ }^{4}$

\section{RESUMO}

Objetivo: descrever o perfil dos trabalhadores acometidos por transtornos mentais relacionados ao trabalho. Método: trata-se de um estudo descritivo, exploratório, com dados secundários, no qual foram analisados os registros de Transtornos Mentais Relacionados ao Trabalho (TMRT) no banco do CEREST Regional Recife, entre os anos de 2015 e 2019. Resultados: contabilizaram-se 310 notificações de modo que a maioria era do sexo feminino $(64,19 \%)$, com faixa etária de $30-40$ anos (45,16\%), branca $(50,32 \%)$, com Ensino Superior completo $(59,68 \%)$ e vínculo empregatício registrado (74,19\%). Acrescenta-se que, dentre os municípios incluídos, Recife obteve o maior número de notificações $(97,74 \%)$ e, em $48,06 \%$ das notificações, houve comunicação de acidente de trabalho. Conclusão: conclui-se que a presença de TMRT interfere nas condições sociodemográficas e no funcionamento do ciclo laboral e familiar, repercutindo negativamente nas atividades diárias e na situação econômica dos trabalhadores.

Descritores: Saúde do Trabalhador; Transtornos Mentais; Enfermagem do Trabalho; Doenças Profissionais; Política Pública.

\footnotetext{
ABSTRACT

Objective: to describe the profile of workers affected by work-related mental disorders. Method: this is a descriptive, exploratory study, with secondary data, in which the records of Work-Related Mental Disorders (WRMD) in the bank of CEREST Regional Recife, between the years 2015 and 2019 were analyzed. Results: 310 notifications were counted, so that the majority were female (64.19\%), aged 30-40 years (45.16\%), white $(50.32 \%)$, with complete college education $(59.68 \%)$, and with registered employment (74.19\%). In addition, among the municipalities included, Recife had the highest number of notifications $(97.74 \%)$ and, in $48.06 \%$ of the notifications, there was communication of a work accident. Conclusion: it is concluded that the presence of WRMD interferes in the socio-demographic conditions and in the functioning of the work and family cycle, having negative repercussions on the daily activities and economic situation of the workers.
} 
Descriptors: Occupational Health; Mental Disorders; Occupational Health Nursing; Occupational Diseases Public Policy

\section{RESUMEN}

Objetivo: describir el perfil de los trabajadores afectados por trastornos mentales relacionados con el trabajo. Método: estudio descriptivo, exploratorio, con datos secundarios, en el que se analizaron los registros de Trastornos Mentales Relacionados con el Trabajo (TMRT) en el banco del CEREST Regional Recife, entre los años 2015 y 2019. Resultados: se contabilizaron 310 notificaciones, por lo que la mayoría eran mujeres $(64,19 \%)$, de edades comprendidas entre los 30 y los 40 años $(45,16 \%)$, de raza blanca $(50,32 \%)$, con estudios superiores completos $(59,68 \%)$ y relación laboral registrada $(74,19 \%)$. Entre los municipios incluidos, Recife tuvo el mayor número de notificaciones $(97,74 \%)$ y en el $48,06 \%$ de las notificaciones, hubo comunicación de accidente laboral. Conclusión: se concluye que la presencia de TMRT interfiere en las condiciones sociodemográficas y en el funcionamiento del ciclo laboral y familiar, repercutiendo negativamente en las actividades diarias y en la situación económica de los trabajadores.

Descriptores: Salud Laboral; Transtornos Mentales; Enfermería del Trabajo; Enfermedades Profesionales; Política Pública.

${ }^{1}$ Centro de Referência em Saúde do Trabalhador/CEREST Regional Recife. Recife(PE), Brasil ORCID:

1 1http: / / orcid.org/0000-0002-2022-7478

2,3,4Universidade Federal de Pernambuco/UFPE. Recife (PE), Brasil.ORCID: 2(B)

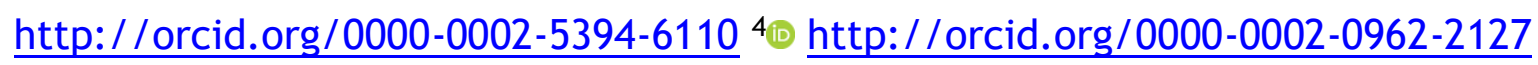

3Universidade Federal Rural de Pernambuco/UFRPE. Recife (PE), Brasil. ORCID: http: / / orcid.org/0000-0001-6318-5872

Artigo extraído do Trabalho de Conclusão de Curso de Especialização em Gestão Pública intitulado “Transtornos mentais relacionados ao trabalho: adoecimento invisível e seus impactos na gestão pública”. Universidade Federal Rural de Pernambuco/UFRPE. 2020.

\section{Como citar este artigo}

Gomes AS, Silva ATCSG, Moura ACP, Pereira DMR. Perfil de trabalhadores acometidos por transtornos mentais relacionados ao trabalho. Rev enferm UFPE on line. 2021;15:e247900 DOI: https:// doi.org/10.5205/1981-8963.2021.247900 
Sabe-se que as exigências determinadas pelo mundo globalizado têm modificado, de forma expressiva, o ambiente de trabalho, tornando-o mais competitivo. Exigem-se, por essas demandas específicas, produtividade, agilidade e dedicação dos trabalhadores. ${ }^{1}$ Gera-se o aumento da prevalência de agravos na saúde mental do profissional pelos esforços para obter êxito nas metas estabelecidas. Eleva-se, por esse fato, a taxa de absenteísmo nos serviços públicos e privados. ${ }^{2}$

Configuram-se, nessa perspectiva, os Transtornos Mentais Relacionados ao Trabalho (TMRT) como a principal causa de adoecimento em trabalhadores, independentemente da inserção no mercado de trabalho. Consideram-se esses transtornos, segundo a Organização Mundial de Saúde (OMS), os mais prevalentes no milênio. Destacam-se, desses, a depressão e a ansiedade. Gera-se, por essas doenças, um custo anual de um trilhão de dólares em perdas de produtividade na economia global. ${ }^{3}$

Podem-se relacionar os transtornos depressivos e de ansiedade aos fatores extrínsecos e intrínsecos do processo laboral e, como exemplo disso, há o assédio moral e sexual, a hierarquização da estrutura organizacional, a carga horária elevada e a dupla jornada de trabalho. ${ }^{4}$

Revela-se que tais fatores são as principais causas de licenças médicas, temporárias ou permanentes, por incapacidade laborativa, tanto no setor privado quanto no público. Detalha-se que, no anterior, os impactos refletem na Previdência Social dos profissionais regidos pela CLT e, no último, esses problemas refletem-se no regime próprio de previdência, a depender da forma de ingresso no serviço público. ${ }^{5}$

Pontua-se que os dados do Anuário Estatístico de Acidentes do Trabalho revelaram que, na região Nordeste, entre os anos de 2015 e 2017, a Previdência Social concedeu 209.197 benefícios, dos quais 143.775 foram registrados com Comunicação de Acidente de Trabalho (CAT) e 7.124 foram reconhecidos como doenças do trabalho. Ressalta-se, além disso, que 743 benefícios relacionados aos TMRT foram deferidos, com predomínio para episódios depressivos, outros transtornos ansiosos, reações de estresse grave e transtorno de adaptação. ${ }^{6}$

Relata-se, pela literatura, no que tange ao acompanhamento desses transtornos, um déficit na resolutividade dos casos. Explica-se que isto ocorre em face da ausência ou do atraso em buscar atendimento especializado, ocasionando repercussões no âmbito financeiro e nos recursos humanos, tendo em vista que o absenteísmo e o presenteísmo laboral impactam diretamente o funcionamento e a qualidade dos serviços, com atraso nas demandas e sobrecarga dos demais funcionários. ${ }^{1,6}$

Torna-se necessária, por sua vez, a integração entre as ações de prevenção, promoção e educação em saúde. Compreendem-se essas como eixo central para estabelecer uma linha de cuidado efetiva e intersetorial que possa acionar os segmentos da sociedade civil, previdência 
social, ministério público, administração pública e saúde, com ações interdisciplinares nos espaços de trabalho. Sublinha-se ainda a importância de registrar as notificações de forma adequada, entendendo a notificação como uma ferramenta indispensável que contribui para a ampliar a detecção precoce dos transtornos supracitados e, consequentemente, delinear ações integrais aos indivíduos. $^{7}$

Justifica-se este estudo, nesse ínterim, como substancial ao investigar o perfil dos trabalhadores e a influência dos dados sociodemográficos sobre o perfil de adoecimento. Faz-se isso com o intuito de compilar os determinantes sociais no processo saúde-doença e elaborar alternativas para a prevenção de agravos relativos aos transtornos relacionados ao trabalho. Formulou-se, para tanto, a seguinte questão condutora: "Qual o perfil dos trabalhadores afastados por transtornos mentais relacionados ao trabalho?".

\section{OBJETIVO}

Descrever o perfil de trabalhadores acometidos por transtornos mentais relacionados ao trabalho.

\section{MÉTODO}

Trata-se de um estudo descritivo, exploratório, com dados secundários obtidos nas notificações de TMRT, emitidas pelo Centro de Referência em Saúde do Trabalhador (CEREST) Regional Recife/SINAN-PE, entre os anos de 2015 e 2019. Informa-se que os estudos descritivos-exploratórios tencionam conhecer as características de um fenômeno para investigar, posteriormente, mais explicações sobre as razões e consequências do fenômeno em questão. ${ }^{8}$

Especifica-se que, embora a região de abrangência do CEREST seja composta por oito municípios (Recife (Sede), Abreu e Lima, Camaragibe, Igarassu, Itamaracá, Itapissuma, Olinda e Paulista), a população de estudo foi constituída por notificações de trabalhadores com TMRT de apenas três localidades, a saber: Camaragibe, Olinda e Recife. Deu-se o critério de escolha após observar que as notificações por TMRT no período descrito foram realizadas apenas nesses municípios.

Elencaram-se como critérios de inclusão nesta pesquisa: trabalhadores notificados no banco de dados do Sistema de Informação de Agravos de Notificação (SINAN); com idade superior a 18 anos e que possuíam o diagnóstico de TMRT, por laudo ou licença médica, de acordo com a Classificação Estatística Internacional de Doenças (CID-10 F) e Problemas Relacionados com a Saúde. Excluíramse as notificações incompletas, duplicadas e os óbitos.

Aponta-se que a principal função da CID, desenvolvida pela OMS, é monitorar a incidência e a prevalência de doenças. Acrescenta-se que isto ocorre por meio de uma padronização universal das doenças, problemas de saúde pública, sinais e sintomas, queixas, causas externas para ferimentos e 
circunstâncias sociais, apresentando um panorama amplo da situação em saúde dos países e suas populações. ${ }^{9}$

Coletaram-se os dados durante os meses de janeiro e fevereiro de 2020. Extraíram-se os dados no sítio eletrônico do SINAN - Recife. Sublinha-se que os dados estão sujeitos à atualização mensal, visto que são preenchidos segundo a notificação dos municípios de abrangência. Priorizaram-se, além disso, os campos de preenchimento obrigatório das fichas de notificação como sexo, raça/cor, faixa etária, escolaridade, município de notificação, situação do trabalhador no mercado de trabatho e emissão da CAT. Digitaram-se e analisaram-se os dados por meio de estatística descritiva (frequências absoluta e relativa), incorporando-os à tabela no Microsoft Office Excel 2007.

Expõe-se que, por tratar-se de um estudo utilizando dados secundários, não houve submissão ao Comitê de Ética em Pesquisa, tendo em vista que a pesquisadora não teve contato com as fichas e as informações expostas não permitiam identificar os indivíduos. Indica-se, entretanto, que foram obedecidos os critérios e princípios éticos e legais presentes na Resolução do Conselho Nacional de Saúde.

\section{RESULTADOS}

Analisaram-se, no período de 2015 a 2019, 310 notificações em TMRT. Observam-se, na tabela 1, a frequência socioeconômica dos casos notificados em Recife e os municípios de abrangência. Destaca-se a predominância de trabalhadoras do sexo feminino, com faixa etária entre 30-40 anos, raça/cor branca, escolaridade superior completa e com vínculo empregatício registrado.

Tabela 1 - Distribuições de notificações de TMRT de acordo com as características socioeconômicas. Recife, PE, Brasil. 2015-2019. n=310

Variáveis

Sexo

Masculino

Feminino

\section{Faixa etária}

n $\%$ 


\section{Raça/cor}

Branca

Preta

Amarela

\section{Escolaridade}

$1^{\mathrm{a}}$ a $4^{\mathrm{a}}$ série incompleta do EF

Ensino Fundamental completo

Ensino Médio incompleto

\section{Situação no mercado de trabalho}

Empregado registrado

\section{Empregado não registrado}

\section{Autônomo}

Servidor público estatutário

Servidor público celetista

Aposentado

Desempregado

Trab. Temporário 
Fonte: Cerest Regional Recife/SINAN-PE, 2015-2019.

Nota-se, com relação à frequência de notificações nos municípios de abrangência, que Recife demonstrou prevalência de $97,74 \%$ dos casos. Verificou-se, além disso, a emissão de CAT em 48,06\% das ocorrências (Tabela 2).

Tabela 2 - Distribuição de notificações de TMRT nos municípios de abrangência e emissão de CAT. Recife, PE, Brasil. 2015-2019. $n=310$

\begin{tabular}{lcc}
\hline Variáveis & $\mathbf{n}$ & $\%$ \\
Municípios & 1 & 0,32 \\
\hline Camaragibe & 6 & 1,94 \\
\hline Olinda & 303 & 97,74 \\
\hline Recife & & 48,06 \\
\hline Emissão de CAT & & 44,52 \\
Sim & 149 & 1,29 \\
\hline Não & 138 & 6,13 \\
Não se aplica & & 4 \\
\hline Ign/Branco & 19 & \\
\hline
\end{tabular}

Fonte: Cerest Regional Recife/SINAN-PE, 2015-2019.

\section{DISCUSSÃO}

Encontrou-se, também, a predominância do sexo feminino em casos de TMRT em um estudo sobre transtornos mentais e comportamentais em trabalhadores do Estado do Piauí. Sabe-se que, historicamente, existe uma procura maior de pessoas do gênero feminino aos serviços de saúde, o que faz com que o número de notificações seja maior. Percebe-se, além disso, que o assédio moral, sexual e as condições de vulnerabilidade concernentes às desigualdades de gênero podem corroborar os achados. ${ }^{11}$

Pode-se relacionar, no que se refere à faixa etária, a prevalência de trabalhadores entre 30 e 40 anos com TMRT ao tempo de trabalho e perfil dos empregos formais, mediados por situações estressantes, sentimentos de desmotivação, irritação, infelicidade e afetividade. ${ }^{12,13}$ Evidenciou-se, com efeito, em um estudo realizado com servidores de um fórum de justiça em Campo Grande 
(MS), que o absenteísmo por doenças foi prevalente em indivíduos com faixa etária de 41-60 anos, fato que corrobora o período de exposição prolongada e o esgotamento físico e mental. ${ }^{14}$

Traduz-se a subjetividade do indivíduo, no tocante à raça/cor, quanto à avaliação desta variável. Atestaram-se, em uma revisão sistemática, resultados contrários aos desta pesquisa, ao afirmar que a prevalência de casos de transtornos mentais é maior em pessoas negras. Pode-se constituir, ao compreender a raça/cor como um determinante social de saúde e adoecimento, esta variável um fator de risco para a ocorrência de transtornos mentais. Verifica-se que ela não se restringe ao aspecto biológico, mas incorpora questões históricas, sociais e econômicas que devem ser consideradas na avaliação de saúde e de qualidade de vida do trabalhador. ${ }^{15}$

Pode-se relacionar a maior frequência de notificações em trabalhadores que possuem instrução de nível superior ao maior nível de exigência e à sobrecarga laboral sucessiva. ${ }^{16}$ Pontua-se que, embora esse achado esteja alinhado à pesquisa realizada com servidores públicos em Santa Catarina, com a prevalência de pós-graduados, ${ }^{17}$ não foi possível estabelecer uma relação de causa e efeito entre a presença de transtornos mentais e o grau de escolaridade, implicando a necessidade de novas investigações acerca dessa problemática. ${ }^{14}$

Observa-se o contrário disso, entretanto, em uma pesquisa conduzida com trabalhadores da atenção básica, em que a escolaridade até o nível técnico ou médio apresentou 1,55 mais chances de desenvolver transtornos mentais comuns, os quais estão relacionados à falta de oportunidades de emprego, às opções de trabalho com condições precárias, à baixa remuneração e ao desconhecimento de direitos. ${ }^{18}$ Torna-se este argumento compatível com a concepção de que quanto maior a escolaridade, melhores são as possibilidades de escolhas na vida laboral, além de influenciar os objetivos, a autoestima e a aquisição de novos conhecimentos, que podem motivar atitudes e comportamentos mais saudáveis. ${ }^{19}$

Indica-se que o predomínio de trabalhadores com registro formal converge com estudos pré$\operatorname{vios}^{20,1}$ e, ainda que exista maior estabilidade e seguridade social nesse modelo de emprego, a Associação Nacional de Medicina do Trabalho (ANAMT) revelou que apenas 18\% das empresas mantêm um programa de assistência à saúde mental dos empregados. ${ }^{21}$

Constata-se, no entanto, que, mesmo sem a devida assistência, os trabalhadores formais conseguem ter acesso aos serviços de referência que efetuam a notificação para o SINAN. ${ }^{20}$ Diz-se outra observação respeito à ausência de vínculo empregatício, que também se faz importante no preenchimento da ficha de notificação. Pode-se associar, de acordo com a Política Nacional Saúde do Trabalhador e da Trabalhadora (PNSTT), o desemprego a transtornos mentais adquiridos durante o tempo de serviço. ${ }^{21}$ 
Realizam-se, desse modo, se o TMRT foi identificado no período em que se realizavam atividades laborativas e, logo após, ocorre a demissão do trabalhador, o registro no SINAN e os demais encaminhamentos para uma ação judicial a fim de reconhecer a doença como profissional.

Percebe-se, quanto aos municípios de abrangência para as notificações de TMRT, que o Recife se destacou. Deve-se isso ao fato de o município conter o Núcleo de Atenção à Saúde do Trabalhador (NAST), que é uma unidade de saúde pública municipal destinada ao atendimento de indivíduos acometidos por doenças e acidentes relacionados ao trabalho nos municípios da abrangência do CEREST Regional - Recife, incluindo Olinda e Camaragibe. Possui-se, além disso, o Recife o maior número de habitantes, comparado aos demais municípios avaliados. ${ }^{22}$

Mostrou-se, em um inquérito descritivo realizado com 210 CERESTs, que o percentual de profissionais capacitados para atendimento em saúde mental foi maior nas regiões Nordeste e Sudeste, equivalendo a 75\% da amostra. Destacaram-se, entre os profissionais envolvidos, o psicólogo, o médico e o enfermeiro, respectivamente. Viu-se, contudo, ainda nesse estudo, que a ausência de ações de assistência para a identificação de diagnósticos e fluxogramas de casos suspeitos de TMRT correspondiam a 53,1\%, o que reforça a urgência em vincular esses profissionais aos programas e intervenções existentes nos dispositivos legais, inclusive, adaptando-os às necessidades locais de cada área. ${ }^{23}$

Trata-se a CAT de um recurso importante para o trabalhador, haja vista que implica o tipo de benefício a ser concedido, oportunizando direitos constituídos de acordo com o vínculo empregatício. Expressa-se, contudo, pelo grande número de respostas negativas na ficha de notificação, um dado preocupante para os trabalhadores. Constata-se que o volume de negações nesse campo converge com o que foi retratado com outro estudo, pois há obrigação legal de gerar a CAT por parte do empregador, sobretudo sob vínculo celetista. Denota-se, pela falha em promover esta comunicação, o descompromisso com a saúde do trabalhador, dificultando a aquisição do benefício governamental. ${ }^{24}$

Pontuam-se, reconhecendo as limitações do estudo, a escassez de notificações dos demais municípios de abrangência da região metropolitana e a falta de detalhes sobre as patologias incluídas nos TMRT.

\section{CONCLUSÃO}

Revela-se que as mulheres com TMRT, com idades entre 30 e 40 anos, raça/cor branca, Ensino Superior e com registro empregatício no município do Recife predominaram neste estudo.

Interfere-se, pela presença desses transtornos na vida do trabalhador, no funcionamento do ciclo laboral e familiar, uma vez que esse tipo de adoecimento repercute negativamente nas atividades diárias e na situação econômica desses indivíduos. Contribui-se, pelo estudo, com informações con- 
sistentes, que podem ser incorporadas no planejamento das ações municipais, para a melhora da saúde do trabalhador.

Observa-se que o número de notificações ainda é pequeno diante da problemática que envolve os TMRT. Acredita-se que cabe aos gestores capacitar e sensibilizar seus trabalhadores quanto à importância da notificação e do preenchimento correto da ficha. Alerta-se que a subnotificação é o maior obstáculo na produção de trabalhos como este, portanto, essa sensibilização viabiliza não só a criação de estratégias de cuidado como também a produção do saber.

\section{CONTRIBUIÇÕES}

Informa-se que todos os autores contribuíram igualmente na concepção do projeto de pesquisa, coleta, análise e discussão dos dados, bem como na redação e revisão crítica do conteúdo com contribuição intelectual e na aprovação da versão final do estudo.

\section{CONFLITO DE INTERESSES}

Os autores declaram não haver conflito de interesses financeiro e/ou de afiliações.

\section{REFERÊNCIAS}

1. Mello IAP, Cazola LHO, Rabacow FM, Nascimento DPG, Pícoli RP. Adoecimento dos trabalhadores da Estratégia Saúde da Família em município da região Centro-Oeste do Brasil. Trab Educ Saúde. 2020; 18(2):e0024390. http://dx.doi.org/10.1590/1981-7746-sol00243

2. Flores LI, Vilela LO, Borelli LM, Júnior EG, Camargo ML. O absenteísmo enquanto indicador para o processo de gestão de pessoas nas organizações e de atenção à saúde do trabalhador. R Laborativa. 2016; 5(2):47-65. Disponível em: https://core.ac.uk/reader/233142468

3. World Health Organization. Mental health in the workplace. Geneva (Swi): WHO; 2019. [cited 2020 Jul 07]. Available from: https://www.who.int/mental_health/in_the_workplace/en/

4. Pascoal FXS. Assédio moral e assédio sexual no ambiente de trabalho. Âmb Jur. 2018; (172). Disponível em: https://ambitojuridico.com.br/edicoes/revista-172/assedio-moral-e-assedio-sexualno-ambiente-de-trabalho/

5. Costa BS, Costa SS, Cintra CLD. Possible impacts of the labor law reform on workers' health. Rev Bras Med Trab. 2018; 6(1):109-17. 10.5327/Z1679443520180097

6. Ministério da Previdência Social (BR). Anuário Estatístico de Acidentes do Trabalho [Internet]. Brasília: 20Disponível em: http:www.previdencia.gov.br/estatísticas.

7. Guimarães LAM, Laudelino Neto A, Massuda Júnior J. Intervenção integrada em saúde mental do trabalhador em uma corporação policial de Campo Grande (MS). Rev Bras Saude Ocup 2020;45:e8. http://dx.doi.org/10.1590/2317-6369000018618 
8. Pintor EAS, Garbin AC. Notificações de violência relacionadas ao trabalho e vigilância em saúde do trabalhador: rompendo a invisibilidade. Rev Bras Saude Ocup. 2019; 44:e20. http://dx.doi.org/10.1590/2317-6369000006918

9. Souza EL, Lyra CO, Costa NDL, Rocha PM, Uchoa AC. Metodologia da pesquisa: aplicabilidade em trabalhos científicos na área da saúde. 2 ed. Natal: EDUFRN, 2019.

10. Organização Mundial da Saúde. CID-10 - Classificação Estatística Internacional de Doenças. 10ª . ed. São Paulo: Edusp; 2008.

11. Fernandes MA, Santos JDM, Moraes LMV, Lima JSR, Feitosa CDA, Sousa LFC. Mental and behavioral disorders in workers: a study on work leave. Rev Esc Enferm USP. 2018; 52:e03396. https://doi.org/10.1590/s1980-220×2017036403396

12. Cacciari P, Haddad MCL, Dalmas JC. Nível de estresse em trabalhadores readequados e readaptados em universidade estadual pública. Texto Contexto Enferm, 2016; 25(2):e4640014. http: //dx.doi.org/10.1590/0104-07072016004640014

13. Vasconcellos LCF, Aguiar L. Saúde do Trabalhador: necessidades desconsideradas pela gestão do Sistema Único de Saúde. Saúde Debate. 2017; 41(113):605-17. https://doi.org/10.1590/01031104201711320

14. França GRMS, Duarte SJH, Cândido MCFS, Moreira AS, Pontes ERJC. Absenteísmo por doença dos servidores do Fórum da Justiça Estadual de Campo Grande (MS). Rev Bras Med Trab. 2019;17(4):582-8. https://dx.doi.org//10.5327/Z1679443520190473

15. Smolen JR, Araújo EM. Raça/cor da pele e transtornos mentais no Brasil: uma revisão sistemática. Ciênc Saúde Colet. 2017; 22(12):4021-30. https://doi.org/10.1590/1413812320172212.19782016

16. Machado LC, Limongi JE. Prevalência e fatores relacionados a transtornos mentais comuns entre professores da rede municipal de ensino, Uberlândia, Minas Gerais, Brasil. Rev Bras Med Trab. 2019;17(3):325-34. https://dx.doi.org//10.5327/Z1679443520190424

17. Baasch D, Trevisan RL, Cruz RM. Perfil epidemiológico dos servidores públicos catarinenses afastados do trabalho por transtornos mentais de 2010 a 2013. Ciênc Saúde Colet. 2017; 22(5):1641-50. https: / /dx.doi.org//10.1590/1413-81232017225.10562015

18. Oliveira AMN, Araújo TM. Situações de desequilíbrio entre esforço-recompensa e transtornos mentais comuns em trabalhadores da atenção básica de saúde. Trab Educ Saúde. 2018; 16(1):24362. http://dx.doi.org/10.1590/1981-7746-sol00100

19. Campos FM, Araújo TM, Viola DN, Oliveira PCS, Sousa CC. Estresse ocupacional e saúde mental no trabalho em saúde: desigualdades de gênero e raça. Cad Saúde Colet. 2020;28(4):579-589. https://doi.org/10.1590/1414-462X202028040559 
20. Carneiro e Cordeiro TMS, Mattos AIS, Castro MCB, Santos KOB, Araújo TM. Notificações de transtornos mentais relacionados ao trabalho entre trabalhadores na Bahia: estudo descritivo, 2007 2012. Epidemiol Serv Saude. 2016; 25(2):363-72. https://doi.org/10.5123/S167949742016000200015

21. Associação Nacional de Medicina do Trabalho. Apenas $18 \%$ das empresas mantêm um programa para cuidar da saúde mental [Internet]. 2018. Disponível em: https://www.anamt.org.br/portal/2018/11/27/apenas-18-das-empresas-mantem-um-programapara-cuidar-da-saude-mental/

22. Gomes AS, Silva ATCSG, Barros MES, Silva RS. Transtornos mentais em bancários atendidos em um serviço de saúde do trabalhador. Rev Enferm Digit Cuid Promoção Saúde. 2020; 5(1):24-28. http://www.dx.doi.org/10.5935/2446-5682.20200006

23. Cardoso MCB, Araújo TM. Atenção aos transtornos mentais relacionados ao Trabalho nas regiões do Brasil. Psicol Soc. 2018; 30:e163746. http://dx.doi.org/10.1590/1807-0310/2018v30163746

24. Zack BT, Ross C, Gouvêa LAVN, Tonini NS. Acidente de trabalho grave: perfil epidemiológico em um município do oeste do Paraná. Saúde Debate. 2020;44(127):1036-52. https: / / doi.org/10.1590/0103-1104202012707

\section{Correspondência}

Adriana dos Santos Gomes

E-mail: dricag_br@yahoo.com.br

Submissão: $17 / 08 / 2020$

Aceito: $13 / 06 / 2021$

Copyright $\odot 2021$ Revista de Enfermagem UFPE on line/REUOL.

(cc) EY Este é um artigo de acesso aberto distribuído sob a Atribuição CC BY 4.0 Creative Commons AttributionShareAlike 4.0 International License, a qual permite que outros distribuam, remixem, adaptem e criem a partir do seu trabalho, mesmo para fins comerciais, desde que lhe atribuam o devido crédito pela criação original. É recomendada para maximizar a disseminação e uso dos materiais licenciados. 